
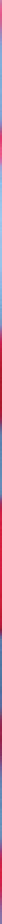

BY ZEEYA MERALI

wen Maroney worries that physicists have spent the better part of a century engaging in fraud.

Ever since they invented quantum theory in the early 1900s, explains Maroney, who is himself a physicist at the University of Oxford, UK, they have been talking about how strange it is - how it allows particles and atoms to move in many directions at once, for example, or to spin clockwise and anticlockwise simultaneously. But talk is not proof, says Maroney. "If we tell the public that quantum theory is weird, we better go out and test that's actually true," he says. "Otherwise we're not doing science, we're just explaining some funny squiggles on a blackboard."

It is this sentiment that has led Maroney and others to develop a new series of experiments to uncover the nature of the wavefunction - the mysterious entity that lies at the heart of quantum weirdness. On paper, the wavefunction is simply a mathematical object that physicists denote with the Greek letter psi ( $\Psi)$ - one of Maroney's funny squiggles — and use to describe a particle's quantum behaviour. Depending on the experiment, the wavefunction allows them to calculate the probability of observing an electron at any particular location, or the chances that its spin is oriented up or down. But the mathematics shed no light on what a wavefunction truly is. Is it a physical thing? Or just a calculating tool for handling an observer's ignorance about the world?

The tests being used to work that out are extremely subtle, and have yet to produce a definitive answer. But researchers are optimistic that a resolution is close. If so, they will finally be able to answer questions that have lingered for decades. Can a particle really be in many places at the same time? Is the Universe continually dividing itself into parallel worlds, each with an alternative version of ourselves? Is there such a thing as an objective reality at all?

"These are the kinds of questions that everybody has asked at some point," says Alessandro Fedrizzi, a physicist at the University of Queensland in Brisbane, Australia. "What is it that is really real?"

Debates over the nature of reality go back to physicists' realization in the early days of quantum theory that particles and waves are two sides of the same coin. A classic example is the double-slit experiment, in which individual electrons are fired at a barrier with two openings: the electron seems to pass through both slits in exactly the same way that a light wave does, creating a banded interference pattern on the other side (see 'Wave-particle weirdness'). In 1926, the Austrian physicist Erwin Schrödinger invented the wavefunction to describe such behaviour, and devised an equation that allowed physicists to calculate it in any given situation ${ }^{1}$. But neither he nor anyone else could say anything about the wavefunction's nature.

\section{IGNORANCE IS BLISS}

From a practical perspective, its nature does not matter. The textbook Copenhagen interpretation of quantum theory, developed in the 1920s mainly by physicists Niels Bohr and Werner Heisenberg, treats the wavefunction as nothing more than a tool for predicting the results of observations, and cautions physicists not to concern themselves with what reality looks like underneath. "You can't blame most physicists for following this 'shut up and calculate' ethos because it has led to tremendous developments in nuclear physics, atomic physics, solidstate physics and particle physics," says Jean Bricmont, a statistical physicist at the Catholic 
An experiment showing that oil droplets can be propelled across a fluid bath by the waves they generate has prompted physicists to reconsider the idea that something similar allows particles to behave like waves.

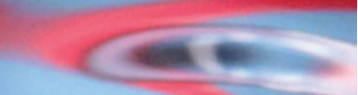
西 西 西 西 西 西

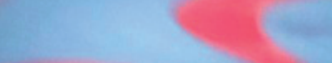

\section{Imagine that a cat is} enclosed in a steel box. And imagine that the box also contains a sample of radioactive material that has a $50 \%$ probability of emitting a decay product in one hour, along with an apparatus that will poison the cat if it detects such a decay. Because radioactive decay is a quantum event, wrote Schrödinger, the rules of quantum theory state that, at the end of the hour, the wavefunction for the box's interior must be an equal mixture of live cat and dead cat.

"Crudely speaking," says Fedrizzi, "in a psiepistemic model the cat in the box is either alive or it's dead and we just don't know because the box is closed." But most psi-ontic models agree with the Copenhagen interpretation: until an observer opens the box and looks, the cat is both alive and dead.

But this is where the debate gets stuck. Which of quantum theory's many interpretations - if any - is correct? That is a tough question to answer experimentally, because the differences between the models are subtle: to be viable, they ace, he says, "there's an overlap and you won't be able to say where it came from". But if you know how many of each type of card is in each deck, you can at least calculate how often such ambiguous situations will arise.

\section{OUT ON A LIMB}

A similar ambiguity occurs in quantum systems. It is not always possible for a single measurement in the lab to distinguish how a photon is polarized, for example. "In real life, it's pretty easy to tell west from slightly south of west, but in quantum systems, it's not that simple," says White. According to the standard Copenhagen interpretation, there is no point in asking what the polarization is because the question does not have an answer - or at least, not until another measurement can determine that answer precisely. But according to the wavefunction-as-ignorance models, the question is perfectly meaningful; it is just that the experimenters - like the card-game player - do not have enough information from that one measurement to answer. As with the cards, it is possible to estimate how much ambiguity can be explained by such ignorance, and compare it with the larger amount of ambiguity allowed by standard theory.

That is essentially what Fedrizzi's team tested. The group measured polarization and other features in a beam of photons and found a level of overlap that could not be explained by

\section{WWE WERE TOLD THAT SUCH EFFECTS CANNOT HAPPEN}

\section{CLASSICALLY, AND HERE WE ARE, SHOWING THAT THEY DO.}

University of Louvain in Belgium. "So people say, let's not worry about the big questions."

But some physicists worried anyway. By the 1930s, Albert Einstein had rejected the Copenhagen interpretation - not least because it allowed two particles to entangle their wavefunctions, producing a situation in which measurements on one could instantaneously determine the state of the other even if the particles were separated by vast distances. Rather than accept such "spooky action at a distance", Einstein preferred to believe that the particles' wavefunctions were incomplete. Perhaps, he suggested, the particles have some kind of 'hidden variables' that determine the outcome of the measurement, but that quantum theories do not capture.

Experiments since then have shown that this spooky action at a distance is quite real, which rules out the particular version of hidden variables that Einstein advocated. But that has not stopped other physicists from coming up with interpretations of their own. These interpretations fall into two broad camps. There are those that agree with Einstein that the wavefunction represents our ignorance - what philosophers call psi-epistemic models. And there are those have to predict essentially the same quantum phenomena as the very successful Copenhagen interpretation. Andrew White, a physicist at the University of Queensland, says that for most of his 20-year career in quantum technologies "the problem was like a giant smooth mountain with no footholds, no way to attack it".

That changed in 2011, with the publication of a theorem about quantum measurements that seemed to rule out the wavefunction-asignorance models ${ }^{2}$. On closer inspection, however, the theorem turned out to leave enough wiggle room for them to survive. Nonetheless, it inspired physicists to think seriously about ways to settle the debate by actually testing the reality of the wavefunction. Maroney had already devised an experiment that should work in principle ${ }^{3}$, and he and others soon found ways to make it work in practice ${ }^{4-6}$. The experiment was carried out last year by Fedrizzi, White and others ${ }^{7}$.

To illustrate the idea behind the test, imagine two stacks of playing cards. One contains only red cards; the other contains only aces. "You're given a card and asked to identify which deck it came from," says Martin Ringbauer, a physicist also at the University of Queensland. If it is a red the ignorance models. The results support the alternative view that, if objective reality exists, then the wavefunction is real. "It's really impressive that the team was able to address a profound issue, with what's actually a very simple experiment," says Andrea Alberti, a physicist at the University of Bonn in Germany.

The conclusion is still not ironclad, however: because the detectors picked up only about one-fifth of the photons used in the test, the team had to assume that the lost photons were behaving in the same way ${ }^{7}$. That is a big assumption, and the group is currently working on closing the sampling gap to produce a definitive result. In the meantime, Maroney's team at Oxford is collaborating with a group at the University of New South Wales in Australia, to perform similar tests with ions, which are easier to track than photons. "Within the next six months we could have a watertight version of this experiment," says Maroney.

But even if their efforts succeed and the wavefunction-as-reality models are favoured, those models come in a variety of flavours - and experimenters will still have to pick them apart.

One of the earliest such interpretations 


\section{WAVE-PARTICLE WEIRDNESS}

When quantum objects such as electrons are fired one by one through a pair of closely spaced slits, they behave like particles: each one hits a screen placed on the far side at exactly one point. But they also behave like waves: successive hits build up a banded interference pattern exactly like that generated by a wave passing through the slits (right). This wave-particle duality is described by a mathematical tool known as the wavefunction.
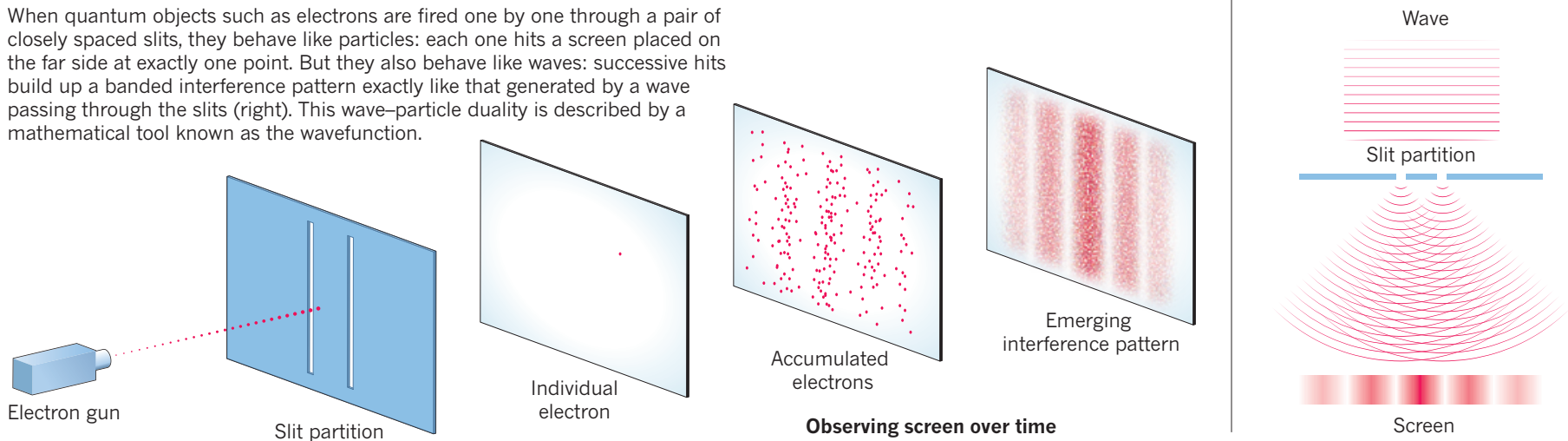

was set out in the 1920s by French physicist Louis de Broglie ${ }^{8}$, and expanded in the 1950s by US physicist David Bohm ${ }^{9,10}$. According to de Broglie-Bohm models, particles have definite locations and properties, but are guided by some kind of 'pilot wave' that is often identified with the wavefunction. This would explain the double-slit experiment because the pilot wave would be able to travel through both slits and produce an interference pattern on the far side, even though the electron it guided would have to pass through one slit or the other.

In 2005, de Broglie-Bohmian mechanics received an experimental boost from an unexpected source. Physicists Emmanuel Fort, now at the Langevin Institute in Paris, and Yves Couder at the University of Paris Diderot gave the students in an undergraduate laboratory class what they thought would be a fairly straightforward task: build an experiment to see how oil droplets falling into a tray filled with oil would coalesce as the tray was vibrated. Much to everyone's surprise, ripples began to form around the droplets when the tray hit a certain vibration frequency. "The drops were self-propelled - surfing or walking on their own waves," says Fort. "This was a dual object we were seeing - a particle driven by a wave."

Since then, Fort and Couder have shown that such waves can guide these 'walkers' through the double-slit experiment as predicted by pilot-wave theory, and can mimic other quantum effects, too ${ }^{11}$. This does not prove that pilot waves exist in the quantum realm, cautions Fort. But it does show how an atomic-scale pilot wave might work. "We were told that such effects cannot happen classically," he says, "and here we are, showing that they do."

Another set of reality-based models, devised in the 1980s, tries to explain the strikingly different properties of small and large objects. "Why electrons and atoms can be in two different places at the same time, but tables, chairs, people and cats can't," says Angelo Bassi, a physicist at the University of Trieste, Italy. Known as 'collapse models', these theories postulate that the wavefunctions of individual particles are real, but can spontaneously lose their quantum properties and snap the particle into, say, a single location. The models are set up so that the odds of this happening are infinitesimal for a single particle, so that quantum effects dominate at the atomic scale. But the probability of collapse grows astronomically as particles clump together, so that macroscopic objects lose their quantum features and behave classically.

One way to test this idea is to look for quantum behaviour in larger and larger objects. If standard quantum theory is correct, there is no limit. And physicists have already carried out double-slit interference experiments with large molecules ${ }^{12}$. But if collapse models are correct, then quantum effects will not be apparent above a certain mass. Various groups are planning to search for such a cut-off using cold atoms, molecules, metal clusters and nanoparticles. They hope to see results within a decade. "What's great about all these kinds of experiments is that we'll be subjecting quantum theory to high-precision tests, where it's never been tested before," says Maroney.

\section{PARALLEL WORLDS}

One wavefunction-as-reality model is already famous and beloved by science-fiction writers: the many-worlds interpretation developed in the 1950s by Hugh Everett, who was then a graduate student at Princeton University in New Jersey. In the many-worlds picture, the wavefunction governs the evolution of reality so profoundly that whenever a quantum measurement is made, the Universe splits into parallel copies. Open the cat's box, in other words, and two parallel worlds will branch out - one with a living cat and another containing a corpse.

Distinguishing Everett's many-worlds interpretation from standard quantum theory is tough because both make exactly the same predictions. But last year, Howard Wiseman at Griffith University in Brisbane and his colleagues proposed a testable multiverse model ${ }^{13}$. Their framework does not contain a wavefunction: particles obey classical rules such as Newton's laws of motion. The weird effects seen in quantum experiments arise because there is a repulsive force between particles and their clones in parallel universes. "The repulsive force between them sets up ripples that propagate through all of these parallel worlds," Wiseman says.

Using computer simulations with as many as 41 interacting worlds, they have shown that this model roughly reproduces a number of quantum effects, including the trajectories of particles in the double-slit experiment ${ }^{13}$. The interference pattern becomes closer to that predicted by standard quantum theory as the number of worlds increases. Because the theory predicts different results depending on the number of universes, says Wiseman, it should be possible to devise ways to check whether his multiverse model is right - meaning that there is no wavefunction, and reality is entirely classical.

Because Wiseman's model does not need a wavefunction, it will remain viable even if future experiments rule out the ignorance models. Also surviving would be models, such as the Copenhagen interpretation, that maintain there is no objective reality - just measurements.

But then, says White, that is the ultimate challenge. Although no one knows how to do it yet, he says, "what would be really exciting is to devise a test for whether there is in fact any objective reality out there at all."

Zeeya Merali is a freelance writer based in London.

1. Schrödinger, E. Phys. Rev. 28, 1049 (1926)

2. Pusey, M. F., Barrett, J. \& Rudolph, T. Nature Phys. 8 , 475-478 (2012).

3. Maroney, O. J. E. Preprint at http://arxiv.org/ abs/1207.6906 (2012).

4. Barrett, J., Cavalcanti, E. G., Lal, R. \& Maroney, O. J.E. Phys. Rev. Lett. 112, 250403 (2014).

5. Leifer, M. S. Phys. Rev. Lett. 112, 160404 (2014).

6. Branciard, C. Phys. Rev. Lett. 113, 020409 (2014).

7. Ringbauer, M. et al. Nature Phys. 11, 249-254 (2015).

8. de Broglie, L. J. Phys. Radium 8, 225-241 (1927).

9. Bohm, D. Phys. Rev. 85, 166-179 (1952).

10.Bohm, D. Phys. Rev. 85, 180-193 (1952)

11.Couder, Y. \& Fort, E. Phys. Rev. Lett. 97, 154101 (2006).

12. Eibenberger, S., Gerlich, S., Arndt, M., Mayor, M. \& Tüxen, J. Phys. Chem. Chem. Phys. 15, 14696-14700 (2013)

13. Hall, M. J. W., Deckert, D.-A. \& Wiseman, H. M. Phys. Rev. X 4, 041013 (2014). 